\title{
Synthesis, characterisation, and the electrochemical sensing behaviour of nicotinic acid hydrazide anchored on graphene oxide (NAHGO)
}

Sreeja B ( $\sim$ Sreeja.pb@christuniversity.in )

Christ University

Jemini Jose

Christ University

Sadasivan Shaji

Universidad Autónoma de Nuevo León

Viswanathan Subramanian

Alagappa University

\section{Research Article}

Keywords: Electro-chemical sensor, Caffeine, Nicotinic acid hydrazide, Electro-oxidation

Posted Date: December 18th, 2020

DOl: https://doi.org/10.21203/rs.3.rs-122247/v1

License: (9) This work is licensed under a Creative Commons Attribution 4.0 International License.

Read Full License 


\section{Abstract}

A simple modified sensor was developed with nicotinic acid hydrazide anchored on graphene oxide (NAHGO), synthesised by ultrasonic assisted chemical route, using hydroxy benzotriazole. Structural and morphologies of NAHGO samples were characterised by Fourier-Transform Infrared spectroscopy (FT-IR), Powder X-ray diffraction (P-XRD), Raman spectroscopy, Scanning electron microscopy (SEM), X-ray photoelectron spectroscopy (XPS) and Thermogravimetric analysis (TGA). Morphology modification of the material provided the route for more electroactive surface, that influenced the electro oxidation of caffeine with increased current. We have demonstrated the electrochemical behaviour of NAHGO on glassy carbon electrode (GCE) for caffeine detection, by employing voltammetric techniques. The influence of scan rate, $\mathrm{pH}$, and concentration on the peak current of caffeine was also studied. The NAHGO sensor was employed for the determination of caffeine in imol plus and energy drink. The detection limit determined was $8.7 \mathrm{nM}$, and the best value reported so far. The results show that NAHGO modified electrodes is one of the best preferences to establish new, efficient, and reliable analytical tools.

\section{Introduction}

Caffeine (1,3,7-trimethyl xanthine) is one of the most consumed purine alkaloid ingredients, as it is a central nervous system stimulant, fatigue alleviator, sharpener of mental function and concentration, taste refresher with unique taste, antioxidant, booster of metabolism and associated health benefits ${ }^{1,2}$. It is commonly found in coffee beans, tea leaves, guarana berries, pharmaceutical drugs, processed foods and energy drinks ${ }^{3}$. The sensible amount of caffeine in medications reduces constipation, depression, headache etc. Like all other drugs, the overdose of caffeine produces anxiety, heartburns, increase of blood pressure, seizures, bone mass loss and cardiovascular diseases ${ }^{4,5}$. The global average consumption of caffeine is $70 \mathrm{mg} /$ person/day ${ }^{6,7}$. On the other hand, the extracts of caffeine act as a pesticide for plants and it has been considered as an indicator for water contamination in sewers ${ }^{8}$. Hence, there is a need for detecting caffeine reliably at lower concentrations, especially, in common beverages, caffeinated food and drugs.

Currently, there are different analytical techniques that are available for the determination of caffeine, such as: high performance liquid chromatography (HPLC), UV spectrometry, capillary electrophoresis, spectrophotometry $9,10,11,12,13$. Though these methods are good to detect caffeine, their complicated, lengthy, time consuming system procedures increase the complexity to monitor the analysis. It makes the requirement of highly sensitive, consistent, simple, and fast methods. In correlation with carbon materials, electrochemical method has proved its quality and efficiency for the fabrication of sensing devices successfully ${ }^{13,14}$. Caffeine has already been electrochemically detected with boron doped diamond electrode, DNA-functionalised single walled carbon nanotubes (SWCNT), attapulgite/nafion, gold nanoparticle, gold-chitosan, multiwalled carbon nanotubes (MWCNT)/diamond like carbon films, glutathione-rGO etc ${ }^{15,16,17,18,19}$. These literature demonstrate that carbon materials has pivotal role for the 
detection of harmful organic molecules like caffeine due to their highly porous structure with large surface area and excellent electronic properties.

Graphene oxide (GO) has got much attention among the carbon nanomaterials owing to its high mechanical strength, thermal and electrical conductivity ${ }^{20}$. Besides, the carboxyl, hydroxy, and epoxy groups create $\mathrm{GO}$ as a desirable platform for further functionalization of molecules ${ }^{21,22}$. Other than functionalization approaches of GO, heteroatom doping also important since it modify the properties of $\mathrm{GO}$ extensively. Among various heteroatom doping, nitrogen doping is widely utilized to employ doping of GO. This is because of the easiness for manipulating GO to improve more active sites for fuel cell, biosensing, adsorption, supercapacitor and solar cell applications ${ }^{23,24}$. Nitrogen doping of GO create different kinds of nitrogen such as, pyrrolic-N, pyridinic- $\mathrm{N}$, and graphitic-N in the $\mathrm{GO}$ lattice which enrich the electronic properties of $\mathrm{GO}^{25,26}$. It has reported that the nitrogen doping changes the spin density and charge distribution on the surface of GO, with the strong interaction between the $\pi$-system of $\mathrm{GO}$ and lone pair of electrons of nitrogen ${ }^{27}$. Hence, we propose a new kind of electrochemical sensor supported on GO and nicotinic acid hydrazide (NAH) for the detection of caffeine. In this study, nicotinic acid hydrazide anchored graphene oxide (NAHGO) was synthesised via ultrasonication-assisted chemical route. The as synthesised NAHGO was analysed by different spectroscopic morphological methods and then studied the electrochemical detection of caffeine. We found that NAHGO showed catalytic activity for the electrochemical oxidation of caffeine.

\section{Results And Discussion}

\subsection{Characterizations of NAHGO}

X-ray photoelectron spectrum of the hybrid NAHGO is done to assess more about the surface composition and chemical states of the elements present in NAHGO. The wide scan spectrum (survey) of $\mathrm{GO}$ and NAHGO are displayed in Fig. 1a. GO shows only the peaks of $\mathrm{C} 1 \mathrm{~s}$ and $01 \mathrm{~s}$, where as NAHGO exhibits the presence of carbon, nitrogen and oxygen, with major prominent peaks at 285.12, 401.23 and $533.41 \mathrm{eV}$ corresponds to $\mathrm{C} 1 \mathrm{~s}, \mathrm{~N} 1 \mathrm{~s}$ and $01 \mathrm{~s}$ respectively. The $\mathrm{C} 1 \mathrm{~s}$ high-resolution spectrum is deconvoluted into four components, and is shown in Fig. 1b. The peaks at 284.5, 285.4, 286.1 and $287.6 \mathrm{eV}$ attributed to $\mathrm{C}-\mathrm{C} / \mathrm{C}=\mathrm{C}$ of graphene oxide framework, $\mathrm{C}-\mathrm{N}, \mathrm{C}-\mathrm{O}$ and $\mathrm{HN}-\mathrm{C}=\mathrm{O}$ bonds. The deconvoluted peaks of $01 \mathrm{~s}$ at 531.5 and $532.9 \mathrm{eV}$, as indicated in Fig. 1c, and are due to the characteristic $\mathrm{C}=\mathrm{O}, \mathrm{C}-\mathrm{O}$ of hydroxyl and epoxy bonds of graphene oxide ${ }^{28,29}$. The individual peaks of N1s at 399.6 and $400.8 \mathrm{eV}$, which are exhibited in Fig. $1 \mathrm{~d}$, attribute to the presence of pyridinic $\mathrm{N}$ and pyrrolic $\mathrm{N}$, respectively. The XRD patterns of GO and NAHGO is presented in Fig. 1e. The intense $2 \theta$ peak at $10.7^{\circ}(001)$ diffraction plane of as prepared GO shows an inter-planar distance $0.83 \mathrm{~nm}$, calculated by the Bragg's equation. In NAHGO, $10.7^{\circ}$ of GO is disappeared and shifted to high $2 \theta$ value, shows the reduction of oxygen containing functional groups and the incorporation of nitrogen atoms onto graphene surface. The Raman spectra of $\mathrm{GO}$ and NAHGO display two major bands as shown in Fig. 1f. The $\mathrm{C}=\mathrm{C}$ stretching vibration of $\mathrm{sp}^{2}$ hybridised carbon systems illustrate the $\mathrm{G}$ band at about $1592 \mathrm{~cm}^{-1}$ and $\mathrm{D}$ band at $1352 \mathrm{~cm}^{-1}$ 
corresponds to the distortions in the edge-centred band structure of carbon framework of $\mathrm{GO}^{29}$. In NAHGO, the positions of these two peaks shift to $1581 \mathrm{~cm}^{-1}$ and $1341 \mathrm{~cm}^{-1}$, confirming the reduction in hybrid $\mathrm{GO}^{30}$. The intensity ratio $\mathrm{I}_{\mathrm{D}} / \mathrm{I}_{\mathrm{G}}$ of $\mathrm{GO}$ and $\mathrm{NAHGO}$ are 0.96 and 1.03 respectively. The increased intensity ratio of $\mathrm{I}_{\mathrm{D}} / \mathrm{I}_{\mathrm{G}}$ in $\mathrm{NAHGO}$, which attributed to defects present after the functionalization of $\mathrm{GO}^{31}$.

The IR spectra of GO and NAHGO are shown in Fig. 2a. The broad peak at $3375 \mathrm{~cm}^{-1}$ is attributed to the stretching vibration of carboxyl $-\mathrm{OH}$ for $\mathrm{GO}$. The $\mathrm{C}=\mathrm{O}, \mathrm{C}-\mathrm{OH}$, and $\mathrm{C}-\mathrm{O}$ of carboxylic and epoxy groups represent peaks at $1721 \mathrm{~cm}^{-1}, 1215 \mathrm{~cm}^{-1}$, and $1030 \mathrm{~cm}^{-1}$, respectively ${ }^{32,33}$. Three new peaks were observed in the spectra of NAHGO. They are at $1625 \mathrm{~cm}^{-1}$, correspond to the amide carbonyl stretch; 1561 $\mathrm{cm}^{-1}$, is due to the N-H of amide band; $1445 \mathrm{~cm}^{-1}$, the C-N stretching of amide bond ${ }^{34,35}$. This is in accordance with XPS, support the nitrogen doping of $\mathrm{GO}$ by nicotinic acid hydrazide. Thermal stability of the GO and NAHGO were investigated by TGA, and are shown in Fig. 2b. The slow degradation of NAHGO with $\mathrm{GO}$ indicates the high stability which acquire by the reduction of $\mathrm{GO}$ and the presence of nitrogen atoms in NAHGO. The XPS data of GO and NAHGO also supports the same. The GO and NAHGO were examined by SEM to understand more about the morphology and microstructure of these compounds. As seen in Fig. 2c-d, GO demonstrated the extended sheet-like structures and NAHGO results the deformation of sheet GO owing to the functionalization of NAH on GO. In NAHGO, the small tube like structures are distributed among the layered sheets of GO. The incorporation of nitrogen moieties on $\mathrm{GO}$ delaminate the sheet structure leads to more voids with crumpled structure. This justifies the significant disorder in the graphitic structure on NAHGO.

\subsection{Electrochemical activity of NAHGO}

The surface area of GO and NAHGO modified working electrodes were probed using 0.001Molar $\mathrm{K}_{4} \mathrm{Fe}(\mathrm{CN})_{6}$ in $0.1 \mathrm{Molar} \mathrm{KCl}$ at scan rate of $50 \mathrm{mV} \mathrm{s}^{-1}$ in potential window of -0.2 to $0.8 \mathrm{~V}$, and are shown in Fig. 3a. The poor electrochemical response of $\mathrm{K}_{4} \mathrm{Fe}(\mathrm{CN})_{6}$ in $0.1 \mathrm{M} \mathrm{KCl}$ at bare GCE implies slow transfer of electrons at the surface. The slight increase of redox peak currents at GCE modified NAHGO than GO possibly owing to fast electron transfer and the large electroactive surface area at NAHGO. This is effective to offer more active sites for caffeine oxidation. The scan rates of NAHGO in a mixture of 0.001 Molar $\mathrm{K}_{4} \mathrm{Fe}(\mathrm{CN})_{6}$ and $0.1 \mathrm{Molar} \mathrm{KCl}$ and plots of anodic current (Ipa) and cathodic current (Ipc) vs square root of the scan rate $\left(\mathrm{u}^{1 / 2}\left(\mathrm{mVs}^{-1}\right)^{1 / 2}\right.$ (Fig S1 and S2a and b) were observed. The electrochemically active surface areas of all the GCE modified electrodes were calculated with the individual slopes of the plot using Randles Sevick equation

$I_{p}=\left(2.69^{\prime} 10^{5}\right) n^{3 / 2} D^{1 / 2} u^{1 / 2} A C^{*}$

which relates the peak current of the active species with surface area for a reversible process ${ }^{36}$. $I_{p}$ refers to the anodic peak current, $n$ is the total number of electrons transferred $(n=1), A$ is the effective surface area of the electrode, $\mathrm{D}$ is the diffusion coefficient of $\mathrm{K}_{4} \mathrm{Fe}(\mathrm{CN})_{6}, \mathrm{C}^{*}$ is the concentration of $\mathrm{K}_{4} \mathrm{Fe}(\mathrm{CN})_{6}$ and $U$ is the scan rate. The calculated electroactive surface areas for bare GCE, GO, and NAHGO modified 
electrodes are $0.078 \mathrm{~cm}^{2}, 0.126 \mathrm{~cm}^{2}$ and $0.29 \mathrm{~cm}^{2}$, respectively. NAHGO seemed to provide an enhanced active surface area presumably owing to the defects in $\mathrm{GO}$ with electron rich nitrogen moieties ${ }^{37}$.

\subsection{Electrocatalytic characteristics of NAHGO for oxidation of caffeine}

The electrochemical activities of bare GCE, GO, and NAHGO were further investigated in the presence of caffeine. Fig. 3b shows CVs of bare GCE, GO, and NAHGO modified GCE electrodes, at pH 2 with $100 \mu \mathrm{M}$ of caffeine, at a scan rate of $50 \mathrm{mV} \mathrm{s}^{-1}$. The electrocatalytic behaviour of the NAHGO was also examined in the absence and presence of caffeine and is shown in Fig. 3c The occurrence of the caffeine oxidation peak in $1.5 \mathrm{~V}$ indicates the oxidation of caffeine on NAHGO modified GCE which is in accordance with the previous reported results ${ }^{19,38}$. Caffeine has tertiary amine group with three alkyl groups and an amide group in which a carboxyl group is connected to a nitrogen atom. In addition to these groups, an imine, carboxyl, methyl, and alkene groups are also present in caffeine. The carbon-oxygen and carbon-nitrogen covalent bonds make caffeine more polar due to its molecular geometry. The distinct positive and negative parts that are created by the overall molecular dipole of caffeine have strong affinity to the ends of other oppositely charged ends of the NAHGO by intermolecular attractions. Though the three nitrogen atoms of caffeine are methylated (N1, N3, and N7) as seen in Fig. S3, caffeine serves as a hydrogen bond acceptor with the ring nitrogen atom (N9) and carbonyl oxygen atoms (02 and 06) ${ }^{39}$. Therefore the interaction of hydrogen is highly dominated on these positions of caffeine. The effective probes of interactions in NAHGO are epoxide, alcohol, carboxylic acid, pyrrolic, and pyridinic nitrogen moieties ${ }^{40}$. The alcohol and carboxylic acid oxygen containing functional groups and the $\mathrm{NH}$ of pyrrolic nitrogen can act as both hydrogen bond donor and acceptor. In this case, they play the role of hydrogen donor and make electrostatic interactions with caffeine. Hence, the increased electrochemical response of NAHGO arise from the efficient interactions of highly polar functional groups of caffeine and NAHGO, which generate charge accumulations on highly polar functional groups turned out to create electrostatic interactions. These interactions are less in $\mathrm{GO}$ due to the less number of heteroatoms which lead to not as much current response of NAHGO.

\subsection{Effect of supporting electrolytes}

To understand the effect of supporting electrolyte on the cyclic voltammetric behaviour of caffeine, different electrolyte solutions of $\mathrm{pH}$ were used. The effect of $\mathrm{pH}$ on the peak currents and peak potentials of caffeine electro-oxidation (100 $\mu \mathrm{M})$ by NAHGO electrode was studied, and are represented in Fig. $3 \mathrm{~d}$. As the $\mathrm{pH}$ changes from 3 to 8 the oxidation peak current weakens, and strongest current was obtained at $\mathrm{pH}$ 2. In pH 2, the electrostatic interactions are more likely to occur with caffeine as mentioned earlier. So, it was considered as the sensible $\mathrm{pH}$ for further experiments. It is also clear that the peak potential shifted negatively as the increase of $\mathrm{pH}$ value of the supporting electrolyte. The linear dependence of anodic current and anodic peak potential with $\mathrm{pH}$ for caffeine are shown in Fig. $3 e$ and $\mathrm{f}$ respectively. The corresponding regression equations are: $\mathrm{Ipa}=0.214 \mathrm{pH}+0.307(\mathrm{R}=0.9963)$ and $\mathrm{Epa}=2.56-0.0491 \mathrm{pH}$ $(R=0.9955)$. This implies that the electrochemical oxidation occurs with equal number of proton and electron transfer process ${ }^{41}$. In the presence of acidic $\mathrm{pH}$, the exchange of electrons between the $\pi$ bonds 
of graphene sheet and the lone pair of electrons bring the polarization effect at the electrode surface. The interactions of NAHGO with acidic protons are shown in Fig. 4.

Cyclic voltammograms of NAHGO (c) with and without caffeine, (d) at pH values of: 2, 3, 5, 6, 7, 8, in 100 $\mu \mathrm{M}$ of caffeine, at a scan rate of $50 \mathrm{mVs}^{-1}$, Calibration plot of (e) pH vs anodic current (Ipa), and (f) pH vs anodic peak potential (Epa).

\subsection{Effect of scan rate}

In order to obtain more knowledge about the adsorption properties of NAHGO the CVs of $100 \mu \mathrm{M}$ caffeine on NAHGO at various scan rates (Fig. S4) were studied for the electro-oxidation process of caffeine. As displayed in Fig. 5 a the anodic peak current of caffeine increases linearly with the increase of scan rate from 10 to $60 \mathrm{mV} \mathrm{s}^{-1}$. The linear relationship of the oxidation current and the square root of the scan rates present that the electrochemical reactions occur on the proposed electrode is diffusion controlled. The oxidation peak potential (Epa) shifts to more positive potential as the gradual increase of scan rates. Laviron model was used to describe the kinetic parameters of the electrochemical oxidation of caffeine ${ }^{42}$. The linear relationship of anodic oxidation potential with logarithms of scan rate is illustrated by this method. According to Laviron, the correlation between the scan rate and the oxidation peak potential is explained as follows:

$E p a=E^{0}+2.3 R T /[(1-\infty) n F] \$ \log u(2)$

As shown in Fig. 5b, Epa exhibits the linear dependence with logarithms of scan rate (log $u)$ and the equation for linearity is Epa $=1.4326+0.0507 \mathrm{log}$ with $\mathrm{R}^{2}=0.9965$. The electrochemical transmission coefficient of an irreversible process is about 0.4-0.6 and for those processes the value is considered to be $0.5^{43}$. Accordingly, the electronic transmission number ( $\mathrm{n}$ ) of electrochemical oxidation of caffeine is calculated as 3.85 , that is in good agreement with the previous results ${ }^{44}$. The mechanism of electrochemical oxidation of caffeine is shown in Fig. 6 as reported earlier ${ }^{45}$. Substituted uric acid is formed in the first step, by the oxidation of two electrons and two protons at C-8-N-9 position, followed by formation of 4,5 diol of uric acid analogue through the immediate two electron and two proton oxidation.

\subsection{Analytical performance}

In the present work, the differential pulse voltammetric method was found to be a more sensitive method, as it produced better electrochemical performance indicators, than the CV technique. Therefore, in order to achieve the favourable analytical performance using differential pulse voltammetric (DPV) method, the parameters such as, pulse amplitude (from $0.05 \mathrm{~V}$ to $0.15 \mathrm{~V}$ ), pulse width (from 0.005 to $0.5 \mathrm{~s}$ ) and pulse period ( 0.1 to $3 \mathrm{~s}$ ) is optimised. The best oxidation peak of caffeine was recorded as follows: pulse amplitude $0.05 \mathrm{~V}$, pulse width $0.06 \mathrm{~s}$ and pulse period of $0.5 \mathrm{~s}$ with NAHGO modified electrode in $0.1 \mathrm{M}$ $\mathrm{H}_{2} \mathrm{SO}_{4}$ at pH 2 in caffeine. Different concentrations from $0.01 \mu \mathrm{M}$ to $40 \mu \mathrm{M}$ of caffeine solutions were prepared to examine the relationship between the peak current and the concentration of caffeine. Fig. $5 \mathrm{c}$ 
displays the linear increase of the anodic peak current (Ipa) with concentrations of caffeine. The calibration graph (Fig. $5 \mathrm{~d}$ ) for caffeine at NAHGO obeys the linear regression equation:

Ipa $(m A)=6.883+0.0989 \mathrm{C} ; R^{2}=0.9906$. The limit of detection (LOD) of caffeine is calculated to estimate this method for the determination of caffeine using the formula 3.3s/S, where s represents residual standard deviation and $\mathrm{S}$ is the slope of the calibration curve ${ }^{38}$ and the LOD for NAHGO modified electrode is found to be $8.7 \mathrm{nM}$, the best reported so far. Moreover, it is compared with other reported values for the determination of caffeine using electrochemical techniques. They are presented in Table 1 and which shows the effectiveness of the proposed NAHGO sensor.

Table 1 An overview of electrochemical methods for the determination of caffeine

\begin{tabular}{|lllll|}
\hline Modified electrodes & Technique & Linear range $(\mu \mathrm{M})$ & $\begin{array}{l}\text { Detection limit } \\
(\mu \mathrm{M})\end{array}$ & References \\
\hline BQ/CPE & SWV & $500-8000$ & 51.00 & 46 \\
\hline Bi-CNT/CPE & SWV & $51.03-1026$ & 0.182 & 47 \\
\hline DNA/CNT/CPE & SWV & $0.5120-61.70$ & 0.350 & 48 \\
\hline MIP/CPE & DPV & $0.0600-25.00$ & 0.015 & 49 \\
\hline CTAB/GR/GCE & DPV & $0.300-100.0$ & 0.091 & 50 \\
\hline SWCNT/CPE & DPV & $0.250-100.0$ & 0.120 & 51 \\
\hline PT/TiO 2 -GR/GCE & DPV & $25.00-200.0$ & 0.500 & 52 \\
\hline Nitrogen doped graphene & SWV & $0.060-50.00$ & 0.020 & 53 \\
\hline Poly(Alizarin Red S)/GCE & SWV & $0.500-250.0$ & 0.060 & 54 \\
\hline Attapulgite/Nafion/GCE & DPV & $1.000-4.000$ & 0.040 & 16 \\
\hline NAHGO/GCE & DPV & $0.010-40.00$ & 0.008 & This work \\
\hline
\end{tabular}

\subsection{Determination of caffeine in energy drink and pharmaceutical sample}

Finally, in order to estimate the practical application of this method, NAHGO/GCE was used to determine caffeine in imol plus and O'cean one8 energy drink. Energy drink was degasified by ultrasonication for 10 minutes before the analysis and diluted with supporting electrolyte $(100 \mu \mathrm{M})$. The tablet was grounded with mortar to a fine powder, dissolved in supporting electrolyte $(100 \mu \mathrm{M})$ and sonicated for 10 minutes. Subsequently, the appropriate amount of the resulting dispersion was selected, and then was centrifuged. $9 \mathrm{~cm}^{3}$ of $0.1 \mathrm{~mol} \mathrm{~L}^{-1} \mathrm{pH} 2$ solution was added to $1 \mathrm{~cm}^{3}$ of the sample solution and recorded the DPVs (Fig.5e and f). In the mean time to calculate the recovery standard solutions of caffeine is added and it is 
represented in Table S1. The recovery range of 94.9-101.9\% suggests that NAHGO is adequate for practical sensing applications.

\section{Experimental}

\subsection{Materials}

Graphite, potassium permanganate, sulphuric acid (36 Molar), hydrochloric acid (5\%), sodium hydroxide, hydrogen peroxide (30\%), hydroxy benzotriazole, dicyclohexyl carbodiimide, nicotinic acid hydrazide, potassium chloride, potassium ferrocyanide, citric acid, sodium citrate, caffeine powder, potassium dihydrogen phosphate, dipotassium hydrogen phosphate were purchased from Sigma Aldrich. All the reactions were carried out under nitrogen atmosphere with the use of standard Schlenk technique. All the solvents and reagents were of analytical grade, and used without further purification. The O'cean one8 energy drink and imol plus were purchased from a local market and pharmaceuticals respectively. The stock solutions of caffeine $(100 \mu \mathrm{M})$ were prepared fresh each time of experiments. Aqueous solutions of different $\mathrm{pH}$ of buffers were prepared, diluted using millipore water.

\subsection{Synthesis of nicotinic acid hydrazide anchored graphene oxide (NAHGO)}

Graphene oxide (G0) was synthesised from graphite, by modified Hummers method ${ }^{28}$. The nicotinic acid hydrazide anchored graphene oxide (NAHGO) was synthesised by the dispersion of GO $(0.2 \mathrm{~g})$ in $20 \mathrm{~cm}^{3}$ dimethyl formamide (DMF), by ultrasonication method. To the solution, sodium hydroxide pellets $(0.2 \mathrm{~g}$, $5.0 \mathrm{mmol}$ ) were added and the resulting solution was stirred for 60 minutes at room temperature. Consequently, nicotinic acid hydrazide $(0.2 \mathrm{~g}, 1.45 \mathrm{mmol})$, hydroxybenzotriazole $(0.2 \mathrm{~g}, 2.81 \mathrm{mmol})$ followed by dicyclohexylcarbodiimide $(0.2 \mathrm{~g}, 2.66 \mathrm{mmol})$ were added to the above reaction mixture, and stirred for 24 hours in nitrogen atmosphere. The resultant NAHGO powder collected by centrifugation was added to pure DMF and the resulting suspension was again centrifuged to remove the side products. This process was repeated twice with DMF and then with water to remove DMF to give pure NAHGO. The NAHGO prepared was dried overnight at $60^{\circ} \mathrm{C}$. The amide, amine, and pyridinic groups are present in NAHGO. The reaction of amino group of NAH with hydroxyl of carboxylic acid generates another amide bond in NAHGO. The imine can form with the amine group of $\mathrm{NAH}$ and the $-\mathrm{C}=\mathrm{O}$ or $-\mathrm{HC}=\mathrm{O}$ group of $\mathrm{GO}$. The nearby carboxylic acid and hydroxyl groups can undergo esterification reaction followed by the generation of pyrrole ring. Hence we believe that the treatment of NAH in GO create more defects as a result of the reduction of $\mathrm{GO}$ with nitrogen doping. The suggested reactions which occur during the synthesis of NAHGO are shown in Fig. 7.

\subsection{Characterisation techniques}

The characteristic functional groups of different samples were recorded using an Agilent technologies FTIR spectrometer in the range 400-4000 $\mathrm{cm}^{-1}$. Powder X-ray Diffraction (P-XRD) patterns were carried out 
in a Brucker AXSD8 Advance using Ni-filtered Cu-K $\mathrm{K}_{\mathrm{a}}$ X-ray source $\left(\lambda=1.5406 \mathrm{~A}^{\circ}\right)$, with a scan speed of $2^{\circ}$ $\mathrm{min}^{-1}$. Thermogravimetric analysis (TGA) was recorded in Perkin Elmer STA 6000. The composition and disorders of anchored compounds were analyzed by X-ray photoelectron spectroscopy (Thermoscientific K-alpha surface analyser). Raman spectra of GO and NAHGO were recorded in HORIBA Jobin Yvon Lab RAM HR 800, equipped with a thermoelectrically cooled CCD detector. The morphology and size of GO and NAHGO were recorded on a JEOL Model JSM-6390LV scanning electron microscope. Cyclic voltammetric (CV) and differential pulse voltammetric (DPV) measurements were carried out on a $\mathrm{CHI608E}$ electrochemical work station with a three electrode system, using saturated $\mathrm{Ag} / \mathrm{AgCl}$ acted as the reference electrode, Pt wire as a counter electrode and the modified glassy carbon electrode as the working electrode. The preparation of the NAHGO modified glassy carbon electrode and the different buffers used are given in supporting information.

\section{Conclusion}

In summary, the synthesis and properties of NAHGO for electrochemical detection of caffeine was discussed. Structure and morphologies of NAHGO were studied by IR, P-XRD, Raman, SEM, and TGA. The electrochemical oxidation of NAHGO towards the alkaloid, caffeine was analyzed. NAHGO displayed enhanced electrochemical activity towards the determination of caffeine, with improved detection limit of $8 \mathrm{nM}$. The NAHGO sensor was also employed for the determination of caffeine in Imol plus and O'cean one8 energy drink. The results of electrochemical studies exhibited that the NAHGO can be used as an efficient and reliable analytical tool for caffeine detection.

\section{Declarations}

\section{Declaration of competing interest}

The authors declare that they have no competing interest.

\section{References}

1. Mejia, E. G. \& Ramirez-Mares, M. V. Impact of caffeine and coffee on our health. Trends Endocrin. Met. 25, 489-492 (2014).

2. Juliana, D. \& Farah, A. Caffeine Consumption through Coffee: Content in the Beverage, Metabolism, Health Benefits and Risks. Beverages 5, 37 (2019).

3. Durrant, K. L. Known and Hidden Sources of Caffeine in Drug, Food, and Natural Products. J. Am. Pharm. Assoc. 42, 625-637 (2002).

4. Robin, P. Oliver, J-K. Paul, R., Jonathan, A-F., Peter. C-H. \& Julie, P. Coffee consumption and health: umbrella review of meta-analyses of multiple health outcomes. BMJ 359, j5024 (2017).

5. Heaney, R. P. Effects of caffeine on bone and the calcium economy. Food Chem. Toxicol. 40, 12631270 (2002). 
6. Poiger, I. J. B. T., Müller, M. D. \& Buser, H. R. Caffeine, an Anthropogenic Marker for Wastewater Contamination of Surface Waters. Environ. Sci. Technol. 37, 691-700 (2003).

7. Senta, I., Lor, E. G., Borsotti, A., Zuccato, E. \& Castiglioni, S. Wastewater analysis to monitor use of caffeine and nicotine and evaluation of their metabolites as biomarkers for population size assessment. Water Research 74, 23-33 (2015).

8. Brodin, T., Piovano, S., Fick, J., Klaminder, J., Heynen, M. \& Jonsson, M. Ecological effects of pharmaceuticals in aquatic systems-impacts through behavioural alterations. Philos Trans $R$ Soc Lond B Biol Sci. 369, 20130580 (2014).

9. Eisele, A. P. P., Valezia, C. F. \& Sartori, E. R. Exploiting the high oxidation potential of carisoprodol on a boron-doped diamond electrode: an improved method for its simultaneous determination with acetaminophen and caffeine. Analyst 142, 3514-3521 (2017).

10. Reskety, A. A., Chamjangali, M. A., Boujnane, M. \& Brajter-Tot, A. High Sensitivity and Fast Oxidation of Caffeine in Coffee and Theophylline at Nanostructured Electrodes. Electroanalysis 28, 1-9 (2016).

11. Oren, T. \& Anık, U. Voltammetric determination of caffeine by using gold nanoparticle-glassy carbon paste composite electrode. Measurement 106, 26-30 (2017).

12. Svorc, L. Determination of Caffeine: A Comprehensive Review on Electrochemical Methods. Int. J. Electrochem. Sci. 8, 5755-5773 (2013).

13. Ramachandran, R. et al. A review of the advanced developments of electrochemical sensors for the detection of toxic and bioactive molecules. Inorg. Chem. Front. 6, 3418-3439 (2019).

14. Kalita, G. \& Tanemura, M. Fundamentals of Chemical Vapor Deposited Graphene and Emerging Applications. In: George K, Athanasios M. Graphene materials-Advanced applications. IntechOpen (2017).

15. Wang, Y., Wei, X., Wang, F. \& Li, M. Sensitive voltammetric detection of caffeine in tea and other beverages based on a DNA functionalized single-walled carbon nanotube modified glassy carbon electrode. Anal. Methods 6, 7525-7531 (2014).

16. Tajeu, K. Y., Ymele, E., Jiokeng, S. L. Z. \& Tonle, I. K. Electrochemical Sensor for Caffeine Based on a Glassy Carbon Electrode Modified with an Attapulgite/nafion Film. Electroanalysis 31, 350-356 (2018).

17. Trani, A., Petrucci, R., Marrosu, G., Zane, D. \& Curulli, A. Selective electrochemical determination of caffeine at a gold chitosan nanocomposite sensor: May little change on nanocomposites synthesis affect selectivity. J. Electroanal. Chem. 788, 99-106 (2017).

18. Silva. T., Zanin, H., Corat, E. J. \& Fatibello-Filho, O. Simultaneous Voltammetric Determination of Paracetamol, Codeine and Caffeine on Diamond-like Carbon Porous Electrodes. Electroanalysis 29, 907-916 (2017).

19. Shehata. M., Azabb, S. M. \& Fekry, A. M. May glutathione and graphene oxide enhance the electrochemical detection of caffeine on carbon paste sensor in aqueous and surfactant media for beverages analysis? Synth. Met. 256, 116122 (2019). 
20. Bottari, G., Ángeles, H. M. \& Wibmer, L. Chemical functionalization and characterization of graphenebased materials. Chem. Soc. Rev. 46, 4464-4500 (2017).

21. Yu, W., Sisi, L., Haiyan, Y. \& Jie, L. Progress in the functional modification of graphene/graphene oxide: A review. RSC Adv 10, 15328-15345 (2020).

22. Jose, J., Rajamani, A. R., Sreekanth, A., Jose, S. P., Sreeja, P. B. \& Peter, S. C. Photophysical and Electrochemical Studies of Anchored Chromium (III) Complex on Reduced Graphene Oxide via Diazonium Chemistry. Appl Organomet. Chem. 33, e5063 (2019).

23. Yadav, R. \& Dixit, C. K. Synthesis, characterization and prospective applications of nitrogen-doped graphene: A short review. J. Sci. Adv. Mater. Devices 2, 141-149 (2017).

24. Jose, J., Jose, S. P., Prasankumar, T., Shaji, S., Saju, P., Sreeja, P. B. Emerging ternary nanocomposite of rGO draped palladium oxide/polypyrrole for high performance supercapacitors. J. Alloys Compd. 855, 157481 (2021).

25. Geng, D. et al. Nitrogen doping effects on the structure of graphene. App/ Surf. Sci. 257, 9193-9198 (2011).

26. Jose, J., Jose, S. P., Abinaya, S., Shaji, S., Sreeja, P. B. Benzoyl hydrazine anchored graphene oxide as supercapacitor electrodes. Mat. Chem. Phy 256, 123666 (2020).

27. Cai, Z. et al. Synthesis of Nitrogen-Doped Graphene Quantum Dots at Low Temperature for Electrochemical Sensing Trinitrotoluene, Anal. Chem. 87, 11803-11811 (2015).

28. Verma, S., Aila, M., Kaul, S. \& Jain, S. L. Immobilized oxo-vanadium Schiff base on graphene oxide as an efficient and recyclable catalyst for the epoxidation of fatty acids and esters. RSC Adv. 4, 3059830604 (2014).

29. Zhang, Y. et al. Phenolic hydroxyl functionalized partially reduced graphene oxides for symmetric supercapacitors with significantly enhanced electrochemical performance. J. Power Sources 415, 226779 (2019).

30. Jhonsi, M. A, Nithya, C. \& Kathiravan, A. Probing electron transfer dynamics of pyranine with reduced graphene oxide. Phys. Chem. Chem. Phys. 16, 20878-20886 (2014).

31. Shanshan, W., Yang, L., Xiaobin, F., Fengbao, Z. \& Guoliang, Z. $\beta$-Cyclodextrin functionalized graphene oxide: an efficient and recyclable adsorbent for the removal of dye pollutants. Front. Chem. Sci. Eng. 9, 77-83 (2015).

32. Kumari, S., Shekhar, A. \& Pathak, D. D. Synthesis and characterization of a Cu(II) Schiff base complex immobilized on graphene oxide and its catalytic application in the green synthesis of propargylamines. RSC Adv. 6, 15340-15344 (2016).

33. Mungse, H. P., Verma, S., Kumar, N., Sain, B. \& Khatri, O. P. Grafting of oxo-vanadium Schiff base on graphene nanosheets and its catalytic activity for the oxidation of alcohols. J. Mater. Chem. 22, 5427-5433 (2012).

34. Chakraborty, S. et al. High yield synthesis of amine functionalized graphene oxide and its surface properties. RSC Adv. 6, 67916-67924 (2016). 
35. Jia, Y. A. N., Gui, C., Jun, C. A. O., Wei, Y., Bang, X. I. E. \& Ming, Y. Functionalized graphene oxide with ethylenediamine and 1,6-hexane diamine, New Carbon Mater. 27, 370-376 (2012).

36. Allen J. B., Larry, R., Faulkner Electrochemical Methods: Fundamentals and Applications, 2nd Edition (2000).

37. Choi, S., Kim, C., Suh, J. M. \& Jang, H. W. Reduced graphene oxide-based materials for electrochemical energy conversion reactions, Carbon Energy 1, 85-108 (2019).

38. Arroyo-Gomez, J. J., Villarroel-Rocha, D., de Freitas-Araújo, K. C., Martínez-Huitle, C. A. \& Sapag, K. Applicability of activated carbon obtained from peach stone as an electrochemical sensor for detecting caffeine. J. Electroanal. Chem. 822, 171-176 (2018).

39. Tavagnacco, L., Schnup, U., Mason, P. E., Marie-Louise, S., Cesàro, A. \& Brady, J. W. Molecular Dynamics Simulation Studies of Caffeine Aggregation in Aqueous Solution. J. Phys. Chem. B 115, 10957-10966 (2011).

40. Hezarkhani, M. \& Ghadari, R. Exploration of the Binding Properties of the Azo Dye Pollutants with Nitrogen-Doped Graphene Oxide by Computational Modeling for Wastewater Treatment Improvement. Chemistry Select 4, 5968-5978 (2019).

41. Wang, Y., Ouyang, X., Ding, Y., Liu, B., Xua, D. \& Liao, L. An electrochemical sensor for determination of tryptophan in the presence of DA based on poly(L-methionine)/graphene modified electrode. RSC Adv. 6, 10662-10669 (2016).

42. Wang, Y, Dinga, Y., Lia, L. \& Huc, P. Nitrogen-doped carbon nanotubes decorated poly (L-Cysteine) as a novel, ultrasensitive electrochemical sensor for simultaneous determination of theophylline and caffeine. Talanta 178, 449-457 (2018).

43. Pelossof, G., Tel-Vered, R., Shimrona, S. \& Willne, I. Controlling interfacial electron transfer and electrocatalysis by $\mathrm{pH}$ or ion-switchable DNA monolayer-modified electrodes. Chem Sci 4, 1137-1144 (2013).

44. Spataru, N., Sarada, B. V., Tryk, D. A. \& Fujishima, A. Anodic Voltammetry of Xanthine, Theophylline, Theobromine and Caffeine at Conductive Diamond Electrodes and Its Analytical Application. Electroanalysis 14, 721-728 (2002).

45. Silva, A. R. L., Araújo, D. M., Silva, E. B. S., Vieira, R. S., Monteiro, N. K. V. \& Martínez-Huitle, C. A. Understanding the behavior of caffeine on a boron-doped diamond surface: voltammetric, DFT, QTAIM and ELF studies. New J. Chem. 41, 7766-7774 (2017).

46. Aklilu, M., Tessema, M. \& Redi-Abshiro, M. Indirect voltammetric determination of caffeine content in coffee using 1,4-benzoquinone modified carbon paste electrode. Talanta 76, 742-746 (2008).

47. Ly, S. Y. et al. Simultaneous diagnostic assay of catechol and caffeine using an in vivo implanted neuro sensor. Bull. Kor. Chem. Soc. 29, 1742-1746 (2008).

48. Ly, S. Y., Lee, C. H. \& Jung, Y. S. Voltammetric Bioassay of Caffeine using Sensor Implant. Neuromolecular Med. 11, 20-27 (2009).

49. Alizadeh, T., Ganjali, M. R., Zare, M. \& Norouzi, P. Development of a voltammetric sensor based on a molecularly imprinted polymer (MIP) for caffeine measurement. Electrochim. Acta 55, 1568-1574 
(2010).

50. Jun-Yong, S., Ke-Jing, H., Shuai-Yun, W. \& Zhi-Wei, W. Application of cetyltrimethylammonium bromide-graphene modified electrode for sensitive determination of caffeine. Can. J. Chem. 89, 697702 (2011).

51. Habibi, B., Abazari, M. \& Pournaghi-Azar, M. H. A Carbon Nanotube Modified Electrode for Determination of Caffeine by Differential Pulse Voltammetry. Chinese J. Cat, 33, 1783-1790 (2012).

52. Xiao-Qin, X., Ke-Jing, H. \& Chun-Xuan, X. Glassy Carbon Electrode Modified With Poly(taurine)/ $/ \mathrm{TiO}_{2}{ }^{-}$ Graphene Composite Film For Determination Of Acetaminophen And Caffeine. Chem. Ind. \& Chem. Eng. 19, 359-368 (2013).

53. Jiang, L., Ding, Y., Jiang, F., Li, L. \& Mo, F. Electrodeposited nitrogen-doped graphene/carbon nanotubes nanocomposite as enhancer for simultaneous and sensitive voltammetric determination of caffeine and vanillin. Anal. Chim. Acta 833, 22-28 (2014).

54. Filik, H., Avan, A. A., \& Mümin, Y. Simultaneous Electrochemical Determination of Caffeine and Vanillin by Using Poly(Alizarin Red S) Modified Glassy Carbon Electrode. Food Anal. Methods 10, 3140 (2017).

\section{Figures}
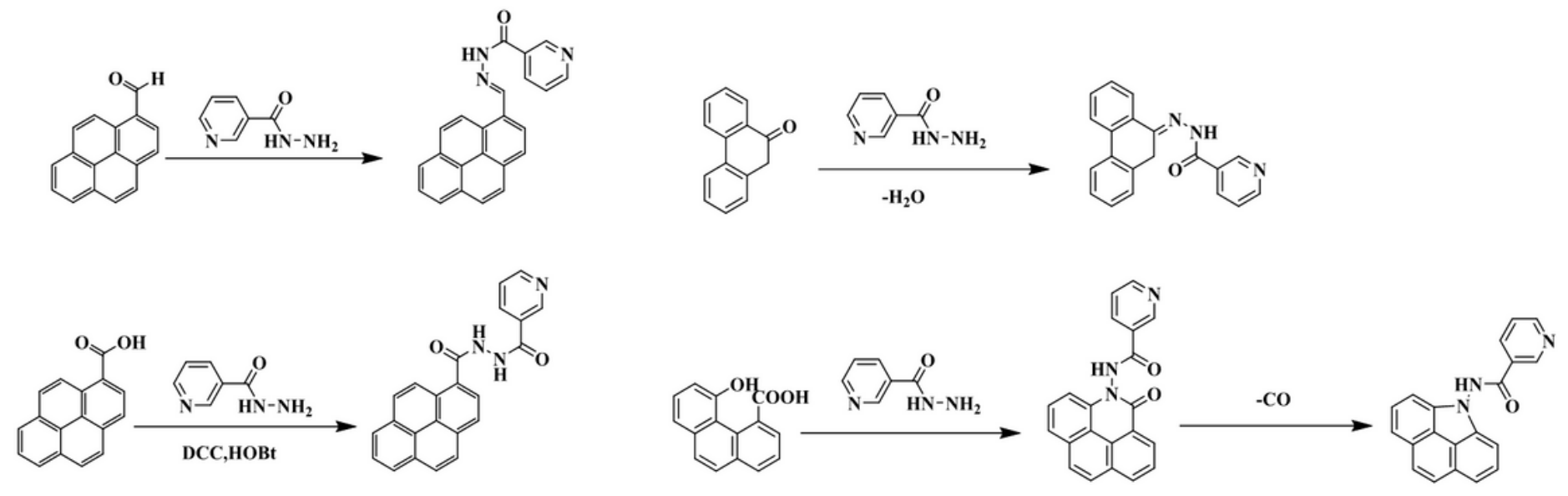

\section{Figure 1}

Reactions occur during the synthesis of NAHGO 
<smiles></smiles><smiles>CCCCCCCCCCCC</smiles><smiles></smiles><smiles>CN1C(=O)N(C)C2(O)N([IH])C(=O)N(C)C2(O)C1=O</smiles>

\section{Figure 1}

Mechanism of the electrochemical oxidation of caffeine at NAHGO 

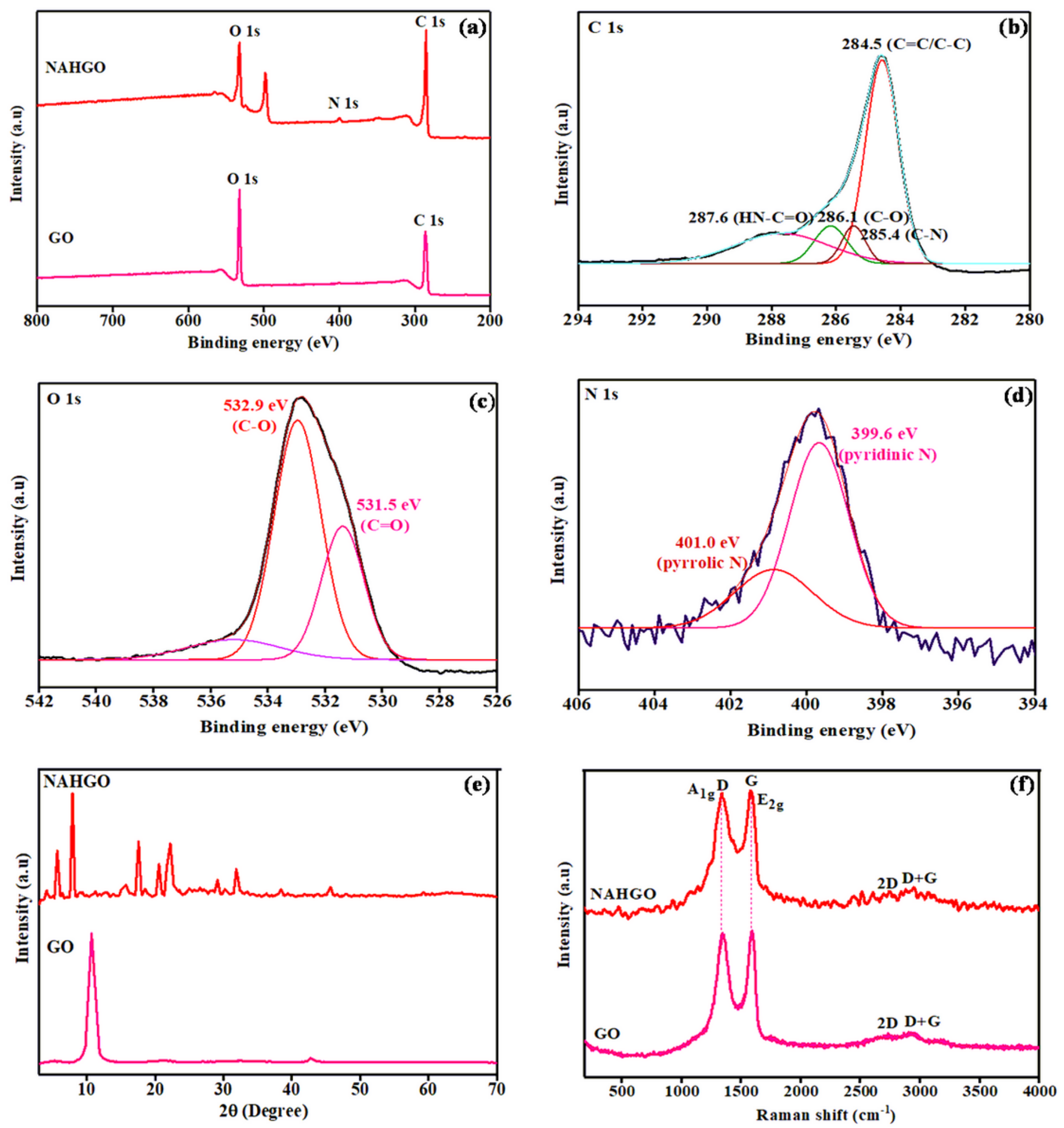

Figure 1

(a) XPS wide scan spectra of GO and NAHGO, deconvoluted peaks of (b) C1s, (c) 01s, and (d) N1s (e) Powder XRD patterns, and (f) Raman spectra of GO and NAHGO 

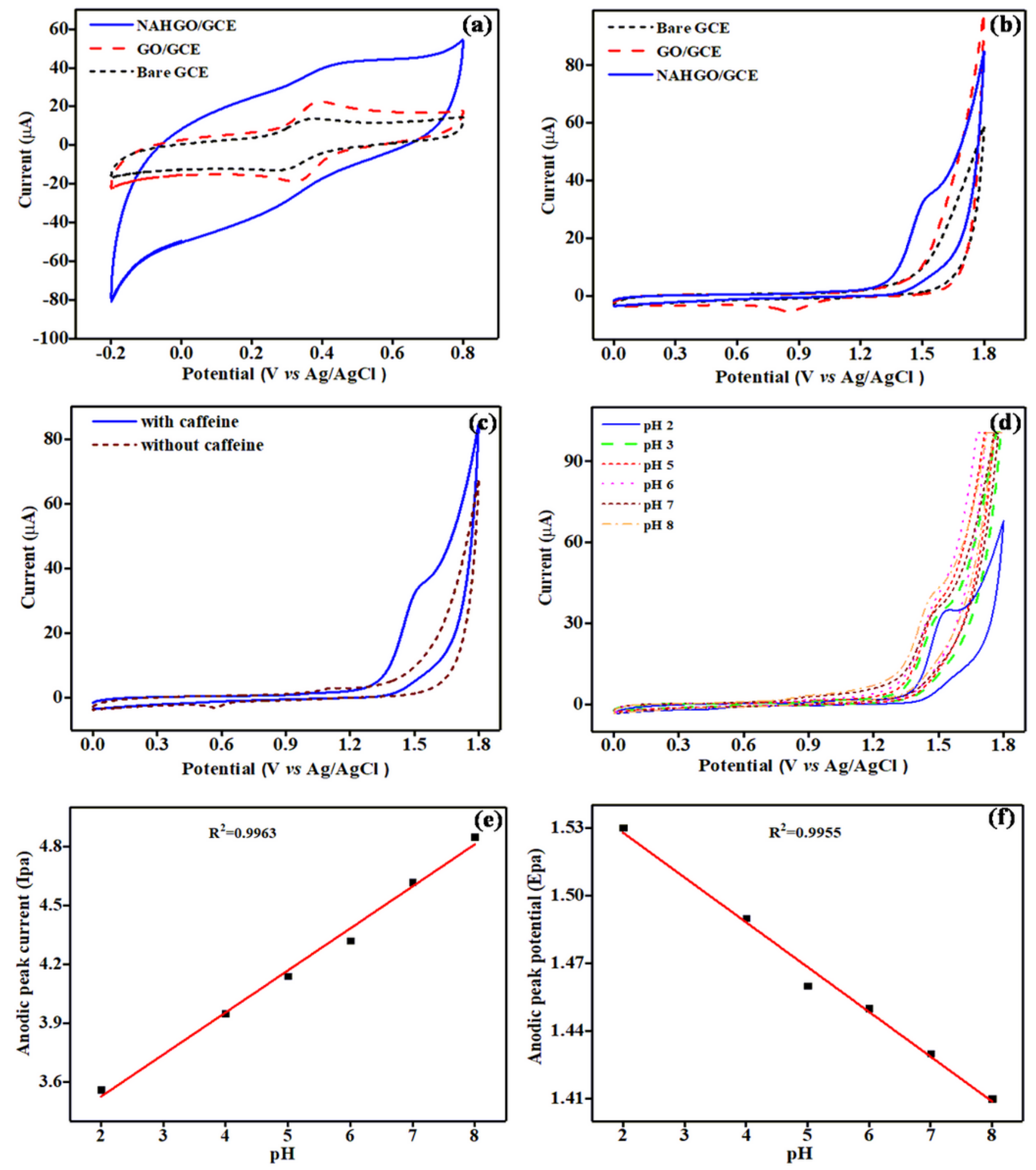

Figure 1

Cyclic voltammograms of the bare GCE, GO and NAHGO (a) in the presence of 0.001 Molar K4Fe(CN)6 in $0.1 \mathrm{Molar} \mathrm{KCl},(\mathrm{b})$ at pH 2 with $100 \mu \mathrm{M}$ of caffeine at a scan rate of $50 \mathrm{mV} \mathrm{s}-1$, Cyclic voltammograms of NAHGO (c) with and without caffeine, (d) at pH values of: 2, 3, 5, 6, 7, 8, in $100 \mu \mathrm{M}$ of caffeine, at a scan rate of $50 \mathrm{mVs}-1$, Calibration plot of (e) pH vs anodic current (Ipa), and (f) pH vs anodic peak potential (Epa). 

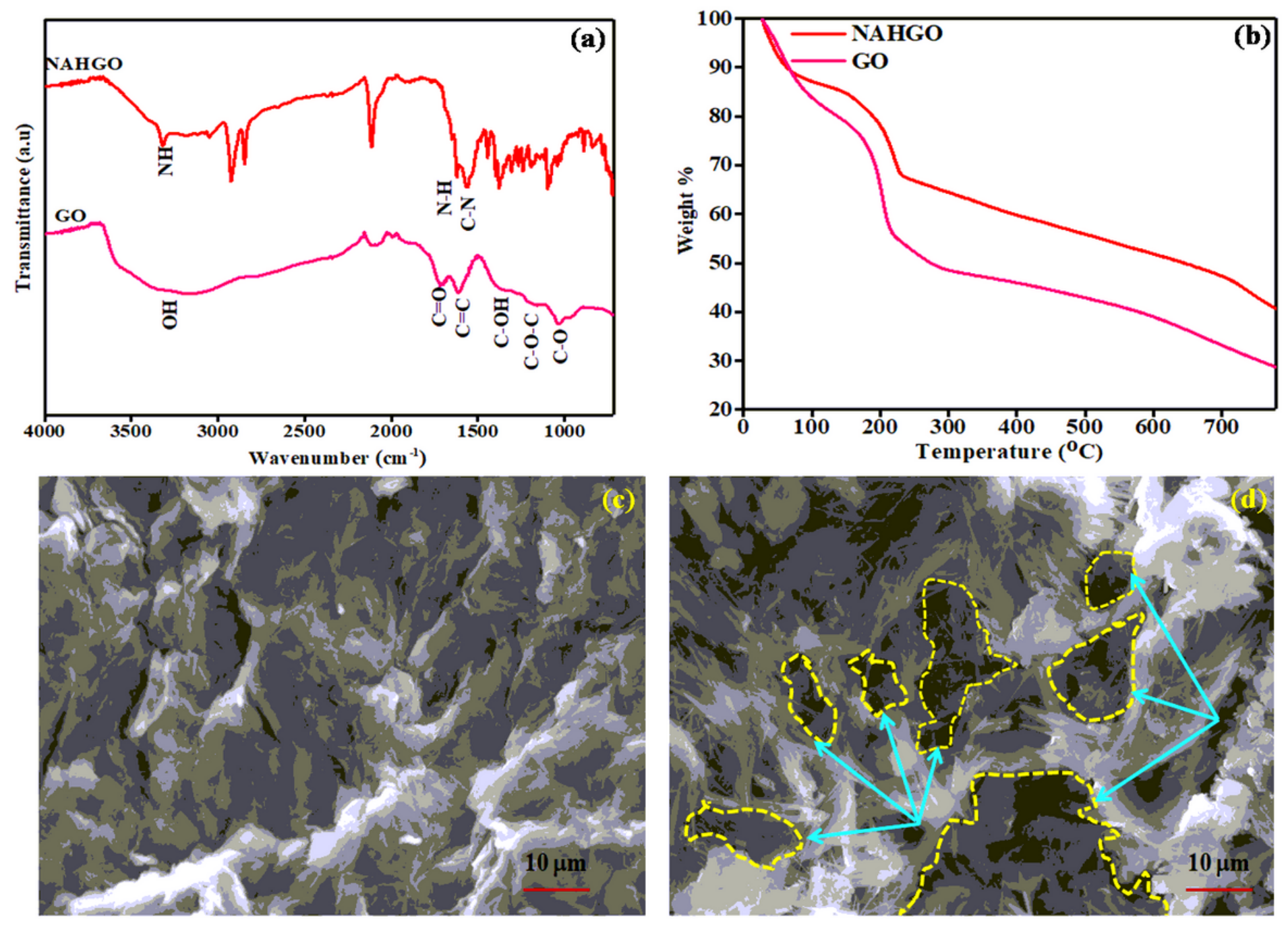

Figure 1

(a) FTIR spectra, (b) Thermogravimetric analysis, (c) and (d) SEM images of GO and NAHGO 

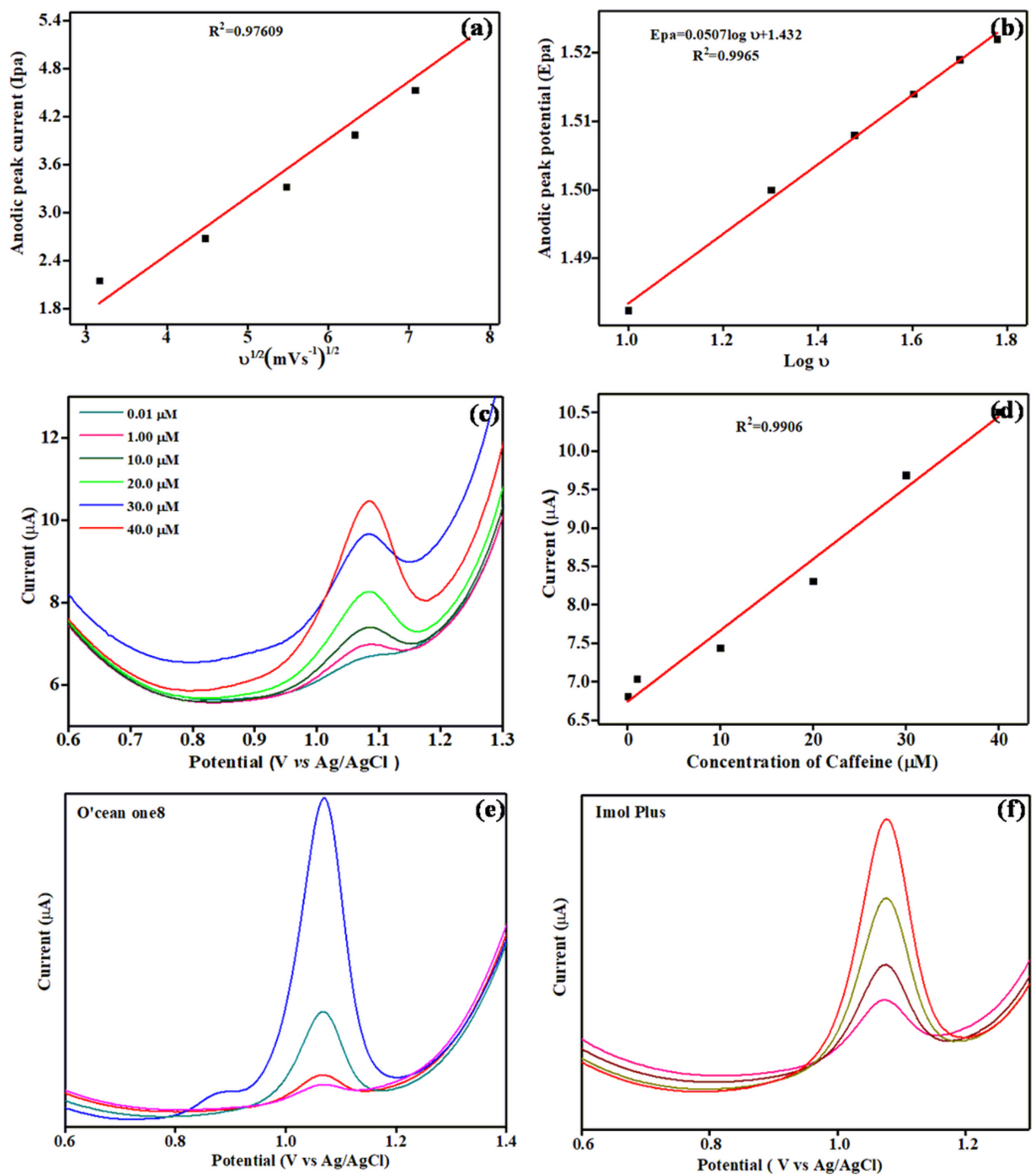

Figure 1

Calibration plot of (a) the square root of the scan rate $\left(\mathrm{U}^{1 / 2}\right)$ vs anodic peak current (Ipa), and (b) logarithm of scan rate (log u ) vs anodic peak potential (Epa), (c) Differential pulse voltammogram (DPV) of the NAHGO modified GCE electrodes at a pH value of 2 with standard additions of caffeine in the order 0.01 $\mu \mathrm{M}, 1.0 \mu \mathrm{M}, 10 \mu \mathrm{M}, 20 \mu \mathrm{M}, 30 \mu \mathrm{M}, 40 \mu \mathrm{M}$ (d) Calibration plot of caffeine concentration ( $\mu \mathrm{M}$ ) vs anodic 
peak current (Ipa), Differential pulse voltammogram of the NAHGO in (e) O'cean one8 energy drink and (f) imol plus.

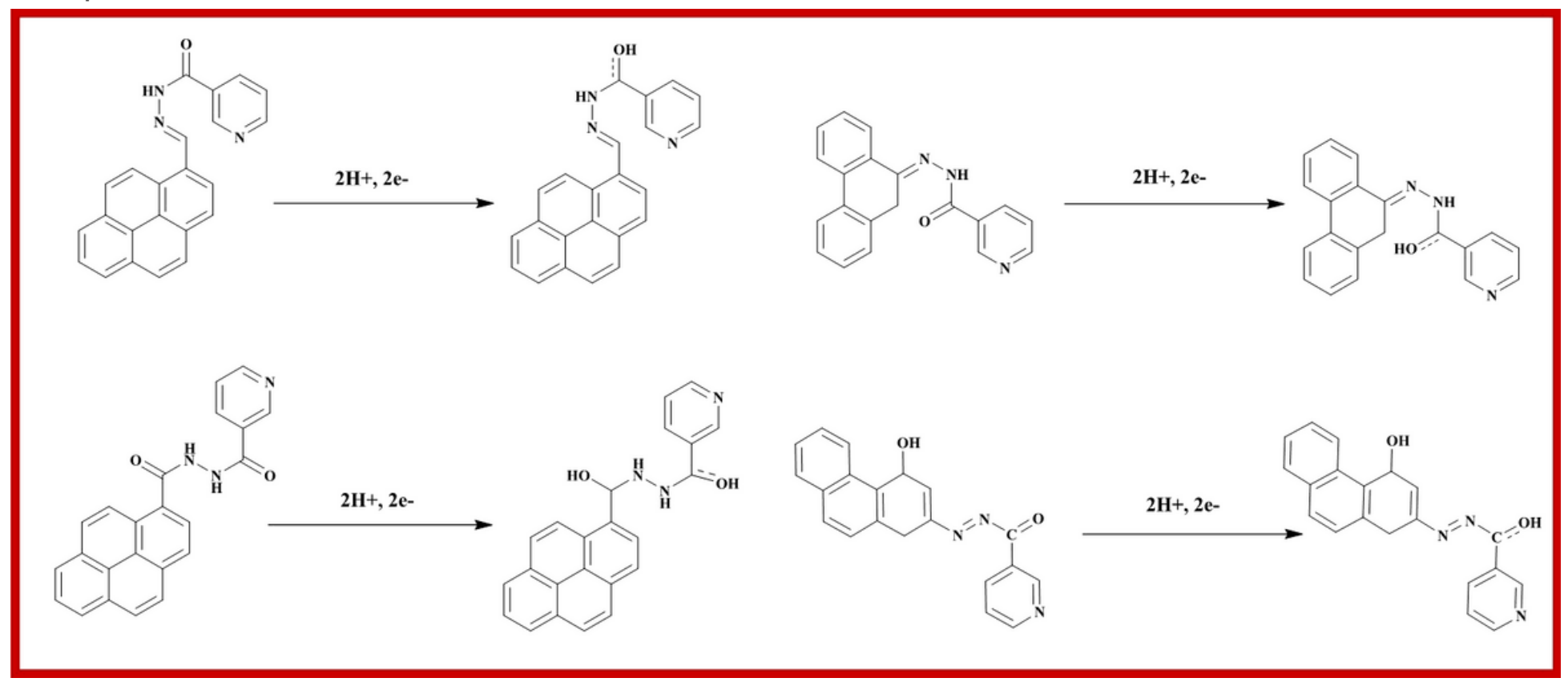

\section{Figure 1}

The interactions of NAHGO at pH 2

\section{Supplementary Files}

This is a list of supplementary files associated with this preprint. Click to download.

- GraphicalAbstract.png

- SciReportsupportinginfoDec8.doc 